\title{
Intergroup Trial C10403: A Pediatric Treatment Approach to Improve Outcomes in Adolescents and Young Adults with Acute Lymphoblastic Leukemia
}

\author{
Katherine Breitenbach, RN, MSN, APN, and Wendy Stock, MD \\ Official trial name: An Intergroup Phase II Clinical Trial for Adolescents and Young Adults \\ with Untreated Acute Lymphoblastic Leukemia (ALL) \\ ClinicalTrials.gov Identifier: NCT00558519 www.clinicaltrials.gov/ct2/show/NCT00558519 \\ CALGB Study Chair: Wendy Stock, MD \\ CALGB Radiation Oncology Co-Chair: John Grecula, MD \\ ECOG Study Co-Chair: Selena Luger, MD \\ SWOG Study Co-Chair: Anjali Advani, MD
}

$\mathbf{O}$ VERALl SURVIVAl AMONG CHILDREN with acute lymphocytic leukemia (ALL) has dramatically increased since the 1970s, with cure rates approaching $85 \% .{ }^{1}$ In comparison, poorer outcomes are found in adolescents and young adults (AYAs, those older than 15 years of age). The rate of ALL-related mortality in 15-24 year olds is greater than any other 10-year age group. ${ }^{2}$ ALL is relatively rare among the AYA population, whereas it is the most commonly diagnosed leukemia in childhood, accounting for $75 \%$ of leukemias diagnosed in pediatric patients. ${ }^{3}$

The biology of this disease also changes with respect to age. Favorable cytogenetics such as hyperploidy and the TELAML1 translocation at $\mathrm{t}(12 ; 21)$ are expressed in approximately a quarter of ALL cases diagnosed in childhood, but decrease dramatically in incidence among the AYA population. ${ }^{4,5}$ T-cell immunophenotype occurs in greater frequency among AYAs than in either younger children or older adults with ALL, and is associated with increased white blood cell count at presentation, higher levels of hemoglobin, and lymphomatous features. ${ }^{6,7}$ Incidence of the Philadelphia chromosome, associated with worse outcomes, increases with age: while it is detected in $25-30 \%$ of adult cases, ${ }^{8} \mathrm{Ph}+$ ALL is present in only a small percentage of AYAs (5-7\%), and is extremely rare among children with ALL. ${ }^{9,10}$ Cytogenetics, immunophenotype, leukocyte count at presentation, and time to achieve complete remission are all prognostically significant factors in ALL. However, age may be the most significant factor pertaining to survival in ALL.

Until recently, minimal outcome data have been available specific to the AYA population. This population is difficult to evaluate and characterize because patients are seen and treated by both pediatric and adult hematologists. Incidence of ALL and enrollment to clinical trials is comparable in children up to the age of 15 years. ${ }^{11}$ In contrast, AYAs enroll to and participate on clinical trials in much smaller numbers. ${ }^{9}$ Because this population represents a small percentage of individuals on clinical trials, they are often included in analyses of either children or older adults. ${ }^{12}$

Adult treatment regimens have historically evolved out of pediatric approaches. However, stark differences exist: pediatric treatment protocols give more frequent doses of nonmyelosuppressive therapy (vincristine, peg-asparaginase, glucocorticoids), central nervous system prophylaxis is given up front and at more frequent intervals, and maintenance therapy is prolonged. In comparison, adult protocols use chemotherapy that is more myelosuppressive with an effort to deliver dose intensity that is tolerated by a broad age range, and overall therapy duration is shorter. The type of therapy AYAs with ALL receive with respect to practitioner (pediatric vs. adult) varies significantly. A retrospective comparison of AYAs who received treatment on Children's Cancer Group (CCG) trials and adult Cancer and Leukemia Group B (CALGB) studies demonstrated that overall survival of patients was superior in AYAs treated on CCG protocols. Although the overall complete response (CR) rate was $90 \%$ in both groups, AYAs treated on adult CALGB trials had only a $34 \%$ event-free survival (EFS) at 7 years, compared to $63 \%$ EFS in demographically similar patients treated on CCG trials. ${ }^{13}$ Similar results are reported in the French FRALLE-93 and LALA-94 trial, as well as reports from Dutch, Italian, and British groups (for review, see Stock, $\left.2010^{14}\right)$.

University of Chicago, Chicago, Illinois. 
In response to the retrospective analyses, a number of studies have been initiated that are pioneering a "pediatricinspired" approach to the treatment of young adults with ALL. One of these studies is the U.S. intergroup trial C10403, developed to examine and describe outcomes among AYAs with newly diagnosed ALL treated using a successful Children's Oncology Group regimen (COG ALL0232) that resulted in a $78 \%$ survival rate for older adolescents aged 16-21 years. ${ }^{15}$ This trial opened to accrual in October 2007, with enrollment currently ongoing at 163 sites nationwide. At present, two-thirds of the estimated 300-participant enrollment have been accrued. Individuals aged 16-39 with newly diagnosed ALL are eligible for participation. This trial will test the feasibility of treating young adults up to the age of 40 with a pediatric regimen, evaluate adherence by patients and adult oncologists, and describe observed toxicities. A component of this trial will analyze outcomes based on demographics, psychosocial characteristics, and pretreatment features of disease that are unique to the AYA population. The results of this trial will be compared with patients up to the age of 29 years who were enrolled and treated by pediatric oncologists on the COG AALL0232 study (now closed to accrual). The goal of this trial is to demonstrate that the adult cancer cooperative groups can deliver a "pediatric" regimen to AYA patients and achieve comparable outcomes. Achievement of this goal would result in a significant improvement in overall survival for young adults with ALL.

\section{References}

1. Smith MA, Seibel NL, Altekruse SF, et al. Outcomes for children and adolescents with cancer: challenges for the twenty-first century. J Clin Oncol. 2010;28:2625-2634.

2. Bleyer A. Older adolescents and young adults with acute lymphoblastic leukemia (ALL) in the United States: from the lowest to highest death rate and number of deaths-more rationale for the CALGB-SWOG-ECOG C10403 trial based on COG AALL0232. J Clin Oncol. 2008;15S:abstract 18034.

3. American Cancer Society. Cancer facts \& figures 2011. Atlanta: American Cancer Society; 2011.

4. Romana SP, Poirel LE, Leconiat M, et al. High frequency of $\mathrm{t}(12 ; 21)$ in childhood B-lineage acute lymphoblastic leukemia. Blood. 1995;86:4263-4269.

5. Moorman AV, Ensor HM, Richards SM, et al. Prognostic effect of chromosomal abnormalities in childhood B-cell precursor acute lymphoblastic leukaemia: results from the UK Medical Research Council ALL97/99 randomised trial. Lancet Oncol. 2010;11:429-438.
6. Heerema NA, Sather HN, Sensel MG, et al. Frequency and clinical significance of cytogenetic abnormalities in pediatric T-lineage acute lymphoblastic leukemia: a report from the Children's Cancer Group. J Clin Oncol. 1998;16:12701278.

7. Larson RA, Dodge RK, Burns CP, et al. A five-drug remission induction regimen with intensive consolidation for adults with acute lymphoblastic leukemia: Cancer and Leukemia Group B study 8811. Blood. 1995;85:2025-2037.

8. Westbrook CA, Hooberman AL, Spino C, et al. Clinical significance of the BCR-ABL fusion gene in adult acute lymphoblastic leukemia: a Cancer and Leukemia Group B study (8762). Blood. 1992;80:2983-2990.

9. Nachman J. Clinical characteristics, biologic features and outcome for young adult patients with acute lymphoblastic leukaemia. Br J Haematol. 2005;130:166-173.

10. Sallan SE. Myths and lessons from the adult/pediatric interface in acute lymphoblastic leukemia. Hematology Am Soc Hematol Educ Program. 2006;2006:128-132.

11. Bleyer A. Adolescent and young adult (AYA) oncology: the first A. Pediatr Hematol Oncol. 2007;24:325-336.

12. Stiller CA, Benjamin S, Cartwright RA, et al. Patterns of care and survival for adolescents and young adults with acute leukemia—a population-based study. Br J Cancer. 1999;79: 658-665.

13. Stock W, La M, Sanford B, et al. What determines the outcomes for adolescents and young adults with acute lymphoblastic leukemia treated on cooperative group protocols? A comparison of Children's Cancer Group and Cancer and Leukemia Group B studies. Blood. 2008;112:1646-1654.

14. Stock W. Adolescents and young adults with acute lymphoblastic leukemia. Hematology Am Soc Hematol Educ Program. 2010;2010:21-29.

15. Nachman JB, La MK, Hunger SP, et al. Young adults with acute lymphoblastic leukemia have an excellent outcome with chemotherapy alone and benefit from intensive postinduction treatment: a report from the Children's Oncology Group. J Clin Oncol. 2009;27:5189-5194.

Address correspondence to: Wendy Stock, MD

Department of Medicine, Section of Hematology Oncology University of Chicago Comprehensive Cancer Center 5841 S. Maryland Ave., MC 2115 Chicago, IL 60637

E-mail:wstock@medicine.bsd.uchicago.edu 